Originalien

Med Klin Intensivmed Notfmed 2022 · 117:209-217 https://doi.org/10.1007/s00063-021-00783-7

Eingegangen: 21. August 2020

Überarbeitet: 19. November 2020

Angenommen: 15. Dezember 2020

Online publiziert: 9 . Februar 2021

(c) Springer Medizin Verlag GmbH, ein Teil von

Springer Nature 2021

\section{Redaktion}

M. Buerke, Siegen

\section{Einleitung}

Infektionen mit SARS-CoV-2-Infektionen sollten aus klinischer Sicht aus unterschiedlichen Perspektiven betrachtet werden, um das Bild der aktuellen Pandemie in Deutschland zu vervollständigen und auf zukünftige Herausforderungen vorbereitet zu sein. Die Klinikperspektive ergänzt bevölkerungsbezogene Sichtweisen (z.B. zu Mortalität, Fallsterblichkeit [„,case fatality proportion“, CFP]) und ermöglicht auf Basis einer Krankenhauspopulation einen direkten Vergleich von SARS-CoV-2-Patienten mit anderen saisonalen Infektionspatienten [9]. Bezogen auf die vorliegenden SARS-CoV-2-Daten weltweit ergibt sich eine CFP von 6,4\% nach WHO (World Health Organisation) bzw. 6,3\% nach Johns Hopkins University. Der minimale Wert lag bei 2,3\% bzw. 2,4\% für die Republik Südkorea, der maximale internationale Wert der CFP bei 20,0\% bzw. 15,5\% für Frankreich [12, 33]. In Deutschland variierten die CFP zwischen minimal 2,6\% in Mecklenburg-Vorpommern und maximal 4,7\% in Bayern. Die SARS-CoV-2-Mortalität/100.000 Einwohner unterschied sich je nach Staat, Bundesland, Landkreis oder Stadt erheblich und streute zwischen 1,2 in Mecklenburg-Vorpommern und 15,7 in Bayern [25]. Es spiegeln sich sowohl der stark differierende lokale Verlauf der Infektionen in den Bundesländern als

M. Englbrecht ist als freiberuflicher Data Scientist tätig.

\author{
M. V. Karg' 1 (D) B. Alber ${ }^{2} \cdot$ C. Kuhn ${ }^{3} \cdot$ K. Bohlinger ${ }^{3} \cdot$ M. Englbrecht ${ }^{4} \cdot$ H. Dormann' \\ 'Zentrale Notaufnahme, Klinikum Fürth, Fürth, Deutschland \\ ${ }^{2}$ Institut für Labormedizin, Klinikum Fürth, Fürth, Deutschland \\ ${ }^{3}$ Gesundheitsamt, Landratsamt Fürth, Zirndorf, Deutschland \\ ${ }^{4}$ Eckental, Deutschland
}

\title{
SARS-CoV-2, Influenza und Norovirus
}

\section{Die Klinikperspektive im Vergleich}

auch unterschiedliche Altersstrukturen oder Risikosituationen wider.

Die Klinikperspektive und damit Fragen über Hospitalisierungsraten, Notaufnahmeprävalenzen und stationäre CFP im direkten Vergleich zu häufigen saisonalen Infektionserkrankungen, wie Influenza oder Norovirusinfektionen, wurde bisher nicht untersucht, obwohl in der Herbst-/Wintersaison große Herausforderungen diesbezüglich zu erwarten sind.

Da die zugrunde liegende Symptomatik einer Infektionserkrankung eine stationäre Behandlung begründet und auch die Indikation zur spezifischen Testung auslöst, soll in der vorliegenden Studie auch die Fragestellung beantwortet werden, wie sich die CFP bei testpositiven von testnegativen hospitalisierten Patienten unterscheiden.

\section{Methodik}

Im Rahmen mehrerer retrospektiver Kohortenanalysen aus 67.000 Krankenhausfällen von Notaufnahmepatienten eines kommunalen Klinikums wurden für die Virusinfektionen SARS-CoV-2, Influenza A/B und Norovirus Genotyp 1 und 2 Hospitalisierungsraten, Notaufnahmeprävalenzen und Fallsterblichkeit („case fatality proportion“, CFP) berechnet.

Für Stadt- und Landkreis mit insgesamt 245.744 Einwohnern stellt die zentrale Notaufnahme des Klinikums den umfassenden Notfallversorger mit

\begin{tabular}{|c|c|c|c|c|c|c|c|}
\hline & $\begin{array}{l}\text { SARS- } \\
\text { CoV-2 }\end{array}$ & $\begin{array}{l}\text { Infl. } \\
2020\end{array}$ & $\begin{array}{l}\text { Infl. } \\
2019\end{array}$ & $\begin{array}{l}\text { Infl. } \\
2018\end{array}$ & $\begin{array}{l}\text { Noro } \\
2020\end{array}$ & $\begin{array}{l}\text { Noro } \\
2019\end{array}$ & $\begin{array}{l}\text { Noro } \\
2018\end{array}$ \\
\hline Tests + Stadtkreis & 824 & 992 & 1038 & 1543 & 135 & 191 & 185 \\
\hline Prävalenz Stadtkreis & $0,34 \%$ & $0,40 \%$ & $0,42 \%$ & $0,63 \%$ & $0,05 \%$ & $0,08 \%$ & $0,08 \%$ \\
\hline $\begin{array}{l}\text { Hospitalisierungsrate/ } \\
100.000 \mathrm{EW}\end{array}$ & 66,1 & 82,0 & 140,4 & 247,3 & 50,6 & 112,7 & 64,9 \\
\hline Todesfälle Stadtkreis & 91 & $x$ & $x$ & $x$ & $x$ & $x$ & $x$ \\
\hline $\begin{array}{l}\text { Fallsterblichkeit (CFP) Stadt- } \\
\text { kreis }\end{array}$ & $11,0 \%$ & $x$ & $x$ & $x$ & $x$ & $x$ & $x$ \\
\hline Hospitalisierungsrate & $19,7 \%$ & $20,3 \%$ & $33,1 \%$ & $39,3 \%$ & $91,9 \%$ & $144,5 \%$ & $85,9 \%$ \\
\hline Test + Klinikum (DRG) & 162 & 201 & 344 & 606 & 125 & 276 & 159 \\
\hline Prävalenz Notaufnahme & $4,1 \%$ & $2,0 \%$ & $2,6 \%$ & $4,0 \%$ & $0,6 \%$ & $1,3 \%$ & $0,8 \%$ \\
\hline Todesfälle Klinikum (DRG) & 48 & 6 & 19 & 39 & 2 & 6 & 2 \\
\hline $\begin{array}{l}\text { Fallsterblichkeit (CFP) Klini- } \\
\text { kum (nach DRG) }\end{array}$ & $29,1 \% *$ & $3,0 \%$ & $5,5 \%$ & $6,4 \%$ & $1,6 \%$ & $2,2 \%$ & $1,3 \%$ \\
\hline
\end{tabular}




\section{Originalien}

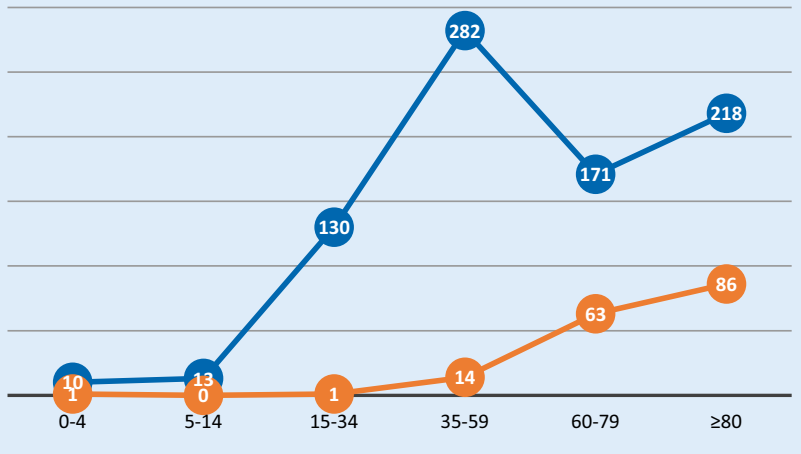

$\longrightarrow$ Stadt/Landkreis $\longrightarrow$ Klinikum

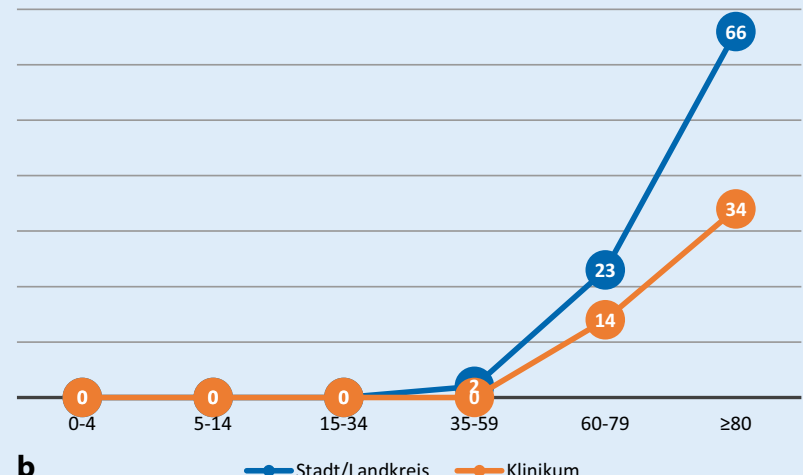

$\longrightarrow$ Stadt/Landkreis $\longrightarrow$ Klinikum

Abb. $1 \Delta$ Altersverteilung und Fallsterblichkeit („,case fatality proportion“ [CFP]) für SARS-CoV-2: positive Einwohner Stadt-/ Landkreis Fürth vs. stationäre SARS-CoV-2-Patienten Klinikum Fürth. a Altersverteilung SARS-CoV-2 - Vergleich Stadt-/ Landkreis vs. Klinikum. b Fallsterblichkeit Stadt-/Landkreis - Klinikum verstorben

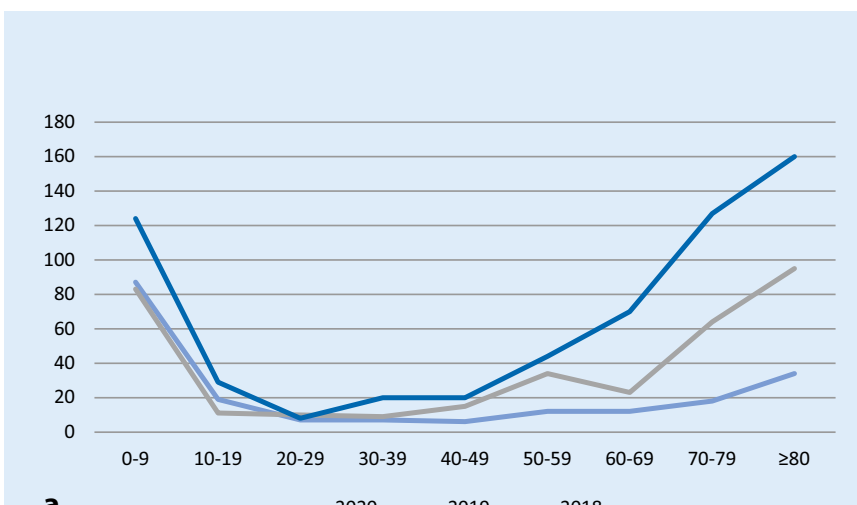

a

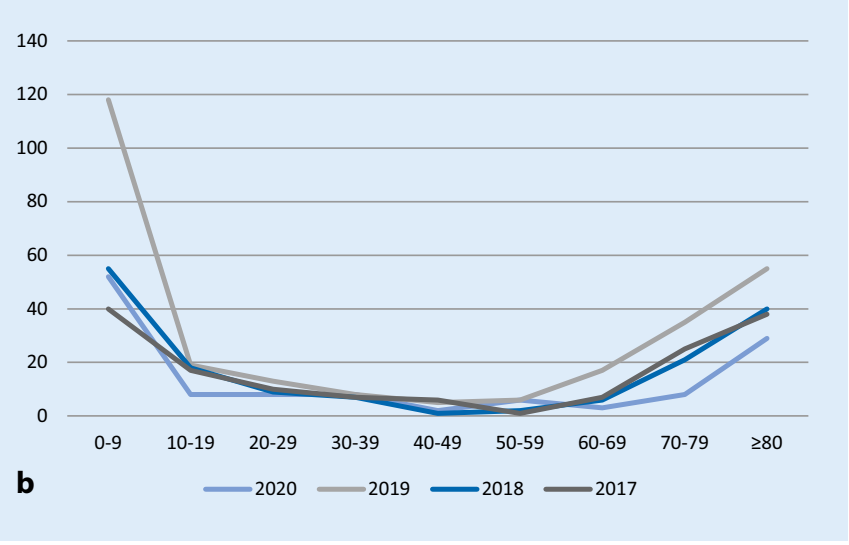

Abb. 2 A Altersstruktur und Fallsterblichkeit („,case fatality proportion“ [CFP]) der stationären Patienten mit Influenza A/B für die Grippesaisons 2018/2019/2020 bzw. der Norovirusinfektionen 2017/2018/2019/2020. a Influenza A/B. b Norovirusinfektionen

jährlich ca. 55.000 Notaufnahmepatienten dar (Bayerisches Landesamt für Statistik, Stand 30.09.2019). Die Altersverteilung der Bevölkerung in Stadtund Landkreis entspricht näherungsweise dem Bundesdurchschnitt [18].

Eingeschlossen in die Analysen wurden alle Personen, die dem Gesundheitsamt der Kommune über einen vom Robert Koch-Institut (RKI) für die jeweilige Infektionssaison definierten Zeitraum aufgrund des Infektionsschutzgesetzes gemeldet wurden. Zusätzlich wurden alle im Klinikum Fürth hospitalisierten SARS-CoV-2-, Influenza A-/B- und Noroviruspatienten, die einem PCR-Test unterzogen wurden oder im Krankenhausinformationssystem (KIS) mittels ICD10-Code (A08, U07.1, J09, J10) über die Jahre 2020, 2019 und 2018 dokumentiert waren, anonymisiert erfasst und ausgewertet anhand von Alter, Geschlecht, Entlassungsstatus Tod und dokumentierte Therapielimitierung zu einer möglichen Wiederbelebung („do not resucitate", DNR) oder invasiven Beatmung („do not intubate“, DNI).

Die PCR-Tests wurden im Klinikum mit einem Gerät der Firma Cepheid (GenXpert [Sunnyvale, CA, USA]) oder von externen kommerziellen oder universitären Laboren erbracht.

Für SARS-CoV-2 wurden alle Patienten bis zum 07.05.2020, für Influenza für die Grippesaisons vom 25.12.2017 bis zum 08.04.2018, vom 07.01.2019 bis zum 07.04.2019 und 06.01.2020 bis zum 22.03.2020 und für Norovirusinfektionen der Zeitraum vom 01.11. bis zum
31.03. eines jeden Jahrs beginnend mit 2017/2018 berücksichtigt [24, 26].

Die Vergleichsanalysen zwischen testpositiven und testnegativen Patienten beziehen sich ausschließlich auf Patienten mit PCR-Testergebnissen, sodass Differenzen zu den Absolutzahlen der Fallauswertungen vorliegen können.

Die Signifikanz und Odds-Ratio zwischen den CFP wurde mittels $\chi^{2}$-Test berechnet. Ein positives Votum zur Durchführung der Studie der Ethikkommission der Friedrich-Alexander-Universität Erlangen-Nürnberg liegt vor. 


\section{Ergebnisse}

\section{SARS-CoV-2 - Stadt- und Land- kreis- vs. Krankenhauspopulation (• Tab. 1)}

In Stadt- und Landkreis wurden 824 Personen ( $0,34 \%$ der Gesamtbevölkerung) positiv auf SARS-CoV-2 registriert, was einer Erkrankungsrate von 335/100.000 Einwohner entspricht. 162 (19,7\%) dieser Patienten wurden im Klinikum hospitalisiert. Das durchschnittliche Alter der positiv getesteten Stadt- und Landkreispopulation lag bei 58,6 Jahre, das der Klinikpopulation bei 75,2 (Min. 0 Jahre [22 Tage], Max. 97 Jahre, $\mathrm{SD} \pm 13,8)$ Jahre. Die Notaufnahmeprävalenz von SARS-CoV-2-Patienten über den Erfassungszeitraum betrug $4,1 \%$, wobei $141(87,0 \%)$ auf Normalstation und 21 auf der Intensivstation behandelt wurden. 48 der hospitalisierten und 91 der Stadt- und Landkreisbewohner verstarben mit SARS-CoV-2Infektion. Das Durchschnittsalter betrug 81,9 Jahre (Min. 64 Jahre, Max. 97 Jahre, $\mathrm{SD} \pm 7,2$ ). Die - Abb. 1a, b zeigt die Altersverteilungen der SARS-CoV-2-Infizierten und Verstorbenen im Vergleich zwischen Kommune und Klinik.

Die CFP für die Stadt- und Landkreispopulation betrug 11,0\%, für die hospitalisierte SARS-CoV-2-Population 29,1\%.

\section{SARS-CoV-2-, Influenza- und Norovirusinfektion - ein Vergleich}

In den Grippesaisons 2020, 2019 bzw. 2018 wurden dem Gesundheitsamt 992, 1038 bzw. 1543 Influenzainfektionen aus Stadt- und Landkreis gemeldet. Die Hospitalisierungsraten betrugen 20,3\% $(n=201), 33,1 \%(n=344)$ und $39,3 \%$ $(n=606)$, die Notaufnahmeprävalenzen 2,0\%, 2,6\% und 4,0\%.

Die CFPs der Influenza-Patienten waren 2020 3,0\%, 2019 5,5\% und in der Grippesaison 2018 6,4\%.

Für die Norovirussaisons 2020, 2019 und 2018 wurden 135, 191 und 185 Personen dem Gesundheitsamt gemeldet. Im Klinikum betrugen die Hospitalisierungsraten $91,9 \%, 144,5 \%$ und $85,9 \%$. Die Prävalenzen waren $0,6 \%, 1,3 \%$ und

Med Klin Intensivmed Notfmed 2022 ·117:209-217 https://doi.org/10.1007/s00063-021-00783-7 ๑) Springer Medizin Verlag GmbH, ein Teil von Springer Nature 2021

\section{V. Karg $\cdot$ B. Alber $\cdot$ C. Kuhn $\cdot$ K. Bohlinger $\cdot$ M. Englbrecht $\cdot$ H. Dormann}

\section{SARS-CoV-2, Influenza und Norovirus. Die Klinikperspektive im Vergleich}

\section{Zusammenfassung}

Hintergrund. Hospitalisierungsraten, Notaufnahmeprävalenzen und Fallsterblichkeiten (CFP) stationärer SARS-CoV-2-Patienten und wie sich diese von anderen pandemischen oder saisonalen Viruserkrankungen, wie Influenza A/B oder Norovirusinfektionen unterscheiden, wurden bisher nicht untersucht. Diese Arbeit gibt einen Überblick aus einer Kommune darüber und vergleicht diesen auch mit den negativ getesteten stationären Verdachtsfällen.

Methoden. Im Rahmen retrospektiver Kohortenanalysen von 67.000 Krankenhausfällen eines Klinikums mit umfassender Notfallversorgung und Meldedaten des regionalen Gesundheitsamts wurden für die Virusinfektionen SARS-CoV-2, Influenza A/B und Norovirus Genotyp 1/2 Hospitalisierungsraten, Notaufnahmeprävalenzen und CFP berechnet.

Ergebnisse. In Fürth (Stadt-/Landkreis) wurden 0,34\% der Bevölkerung, 824 Personen, bis 07.05 .2020 positiv auf SARSCoV-2 getestet, wovon $162(19,7 \%)$ stationär behandelt wurden. 91 der Infizierten verstarben (CFP 11,0\%), davon 48 stationär. In der aktuellen Grippe-/Norovirussaison wurden 992 Einwohner als influenzapositiv und 135 als noroviruspositiv gemeldet, davon 202 (20,3\%) bzw. 125 (91,9\%) stationär behandelt. Die Notaufnahmeprävalenzen waren $4,1 \%, 2,0 \%$ und $0,6 \%$. Die CFP der SARS-CoV-2-, influenza- und noroviruspositiven Krankenhauspopulationen betrugen $29,1 \%, 3,0 \%$ und $1,6 \%$, die der testnegativen Verdachtsfälle $5,9 \%, 4,8 \%$ und $6,9 \%$ bei einer Krankenhausmortalität von 2,1 \% für 2020. Schlussfolgerungen. Bei gleichen Hospitalisierungsraten von SARS-CoV-2- und Influenzapatienten unterschieden sich die CFP massiv, während die CFP der testnegativen Verdachtsfälle aller 3 Infektionserkrankungen sich nicht signifikant unterschieden.

\section{Schlüsselwörter}

COVID-19 · Grippe · Fallsterblichkeit (CFP) . Hospitalisierungsrate · Notaufnahmeprävalenz

\section{SARS-CoV-2, influenza and norovirus infection. A direct epidemiologic comparison}

\section{Abstract}

Background. Hospitalization rates (HR), emergency care prevalence (ECP) and case fatality proportions (CFP) of hospitalized severe acute respiratory syndrome coronavirus 2 (SARS-CoV-2) patients in one municipality have not been studied, including how these differ from other pandemic or seasonal viral diseases, such as influenza $A / B$ or norovirus. These analyses and their comparison with negative-tested hospitalized patients are the purpose of the present study.

Methods. A total of 67,000 cases of hospitalized patients of a tertiary care hospital and data of the regional health institute were analyzed to calculate HR, ECP and CFP of SARS-CoV-2, influenza $A / B$ and norovirus genotype $1 / 2$.

Results. In the city and district of Fürth (Germany), 824 persons (0.34\%) tested SARS-CoV-2-positive through 7 May 2020, 162 of whom (19.7\%) were hospitalized. Furthermore, 48 out of 91 patients died in hospital (CFP 11.0\%). During the flu/norovirus season 2019/2020 992 inhabitants were flupositive and 135 norovirus-positive, of whom $202(20.3 \%)$ and 125 (91.9\%), respectively, were hospitalized. The ECPs were $4.1 \%, 2.0 \%$ and $0.6 \%$. The CFPs of the SARS-CoV-2-, influenza- and norovirus-positive hospital cohort were $29.1 \%, 3.0 \%$ and $1.6 \%$, while CFPs of the negative-tested were $5.9 \%, 4.8 \%$ and $6.9 \%$ with an in-hospital mortality of $2.1 \%$ for 2020 .

Conclusions. While HRs of SARS-CoV-2 and influenza were similar, CFPs differ significantly. CFPs of negative-tested patients were comparable between the three infectious diseases.

Keywords COVID-19 · Flu - Case fatality proportion, CFP. Hospitalisation rates - Emergency care prevalence 


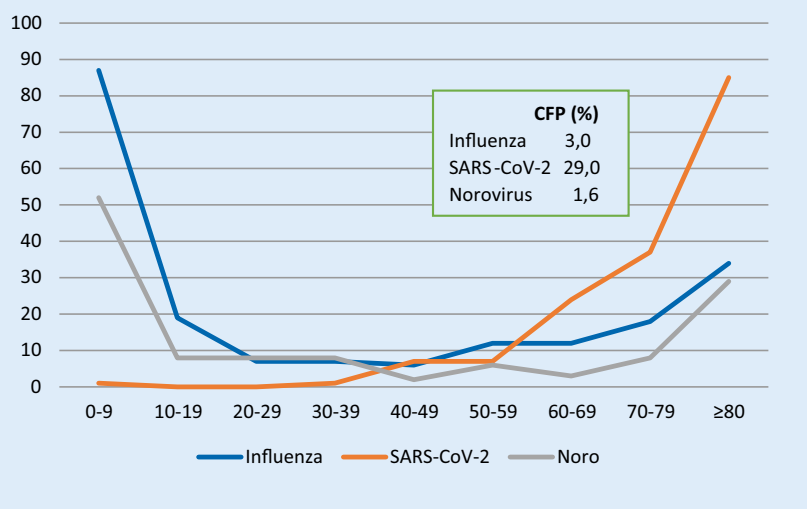

Abb. $3<$ Altersverteilung und Fallsterblichkeit („,case fatality proportion" [CFP]) der stationären Patienten 2020 mit Influenza A/B, SARS-CoV2-bzw. Norovirusinfektion

$0,8 \%$, die CFP lagen bei 1,6\%, 2,2\% und $1,3 \%$.

Die - Abb. 2a, b zeigt die Altersverteilungen der hospitalisierten Influenzaund Noroviruspatienten der letzten 3 Infektionssaisons.

Die Altersverteilung der hospitalisierten Patienten aller 3 Infektionserkrankungen zeigt in höherem Lebensalter einen deutlichen Anstieg. Die erhöhten Patientenzahlen im Kindes- und Jugendalter fehlen bei SARS-CoV-2-Infizierten, sodass erst ab der 4. Lebensdekade ein Anstieg zu verzeichnen ist (• Abb. 3).

Bei der detaillierten Betrachtung der Sterbefälle zeigte sich, dass bei 40 von insgesamt 48 SARS-CoV-2-Patienten und 5 von 6 in 2020 bzw. 12 von 19 in 2019 positiv auf Influenza getesteten Patienten bei Behandlungsbeginn DNR oder DNI als Ausdruck des Patientenwillens im KIS dokumentiert war.

\section{Vergleich der CFP zwischen testpositiven und testnegativen Patienten}

Die - Tab. 2, 3, 4 und 5 zeigen eine zusammenfassende Darstellung der hospitalisierten positiv bzw. negativ getesteten SARS-CoV-2- und Noroviruspatienten 2020, für Influenza zusätzlich 2019. Während die CFP für SARS-CoV-2-positiv Getestete mit 29,0\% deutlich über der CFP der negativ Getesteten mit 5,9\% lag, zeigt sich, dass diese vergleichbar mit der CFP von Influenzapositiven im Jahr 2019 ist. Auffällig ist auch, dass sich die CFP aller negativ getesteten Patienten aller 3 Infektionserkrankungen zwischen $4,8 \%$ und $6,9 \%$ auf dem Niveau der ne- gativ getesteten SARS-CoV-2-Patienten bewegen.

Keiner der 2020 hospitalisierten $\mathrm{Pa}$ tienten wies eine Koinfektion von Influenza, Norovirus und SARS-CoV-2 auf. Bemerkenswert ist auch die Tatsache, dass die CFP der negativ getesteten Influenza- und Noroviruspatienten im Jahr 2020 höher lagen als die CFP der positiv getesteten Patienten.

Die Mortalität der Notaufnahmepatienten insgesamt betrug für Januar bis Mai 2020 4,5\%, für 20193,8 \% bei einer Krankenhausmortalität von $2,6 \%$ und 2,1\% im Vergleichszeitraum.

Die Odds-Ratio für hospitalisierte SARS-CoV-2-Patienten $\mathrm{zu}$ versterben betrug 6,51 (95\%-KI: 4,25-9,98; $p<0,001)$ im Vergleich zu Influenzaund Noroviruspatienten (• Tab. 1). Interessanterweise ergibt sich auch für Patienten mit negativem Norovirustest ein erhöhtes Risiko, an einer Durchfallerkrankung zu versterben, verglichen mit positiv getesteten Noroviruspatienten (6,9\% vs. $1,6 \%)$.

\section{Diskussion}

Bei einer Infektionserkrankung, die einen hohen Anteil asymptomatischer oder symptomarmer Personen verursacht, variieren Prävalenz, bestätigte CFP und bevölkerungsbezogene Mortalität aufgrund unterschiedlicher Testhäufigkeiten erheblich, sodass nur bedingt Rückschlüsse auf Gefährlichkeit und klinischen Verlauf gezogen werden können $[19,35]$. Dennoch ergab die Prävalenz von SARS-CoV-2 für Stadt- und Landkreis in dieser Studie mit $0,34 \%$ einen fast identischen Wert, verglichen mit einer repräsentativen österreichischen Querschnittstudie mit 0,33\% [34], wobei in der Literatur Prävalenzen mit Spitzenwerten von 13-19\% für die „Hotspots“ in Gangelt (Deutschland) oder St. Anton (Österreich) beschrieben werden [21, 31].

In Ergänzung dazu fokussiert unsere Studie die Population der hospitalisierten SARS-CoV-2-Patienten und setzt sie in direkten Vergleich zu Influenza- und Noroviruspatienten der letzten 3 Jahre. Da die Hospitalisierung zumeist symptomgetriggert und auch Ausdruck der Krankheitsschwere ist, werden neben der Hospitalisierungsrate die Notaufnahmeprävalenz und die stationären CFP sowohl von positiv getesteten Patienten als auch von negativ getesteten Verdachtsfällen analysiert.

Hierbei zeigt sich für SARS-CoV-2Patienten eine im Vergleich zu Influenzaoder Noroviruspatienten hochsignifikant erhöhte CFP mit 29,1\%, wohingegen die CFP der testnegativen Verdachtsfälle aller 3 Infektionserkrankungen zwischen 4,8 \% und 6,9\% ähnlich waren (• Tab. 2, 3, 4, 5 und 6) und sogar der für Deutschland publizierten COVID-19-CFP von 4,6\% entsprachen [12,33]. Die Mortalitäten aller Klinikpatienten und der Notaufnahmepatienten lagen mit 2,0-4,5\% nur tendenziell unter den CFP der negativ getesteten SARS-CoV-2- und der positiv oder negativ getesteten Influenza- und Noroviruspatienten. Unsere CFP von 1,6\% für Noroviruspatienten entspricht der von Briel et al. mit 1,5\% [2]. Leider wurden in dessen Studie nicht die testnegativen Patienten ausgewertet, da auffällt, dass für die negativ getesteten Influenza- und v. a. Noroviruspatienten im Jahr 2020 die CFP höher lagen, als für die tatsächlich an Influenza und Norovirus Erkrankten.

Von den 43 influenzanegativ getesteten Patienten wurden 6 auch auf SARSCoV-2 getestet, wobei sich 2 bestätigten. Aufgrund des klinischen Verlaufs in der Frühphase der Pandemie könnte bei 20 weiteren Todesfällen postuliert werden, dass eine SARS-CoV-2-Infektion nicht ausgeschlossen werden kann. Bei 3 Todesfällen lag eine RSV-Infektion vor. In keinem einzigen Fall wurde eine Koinfektion diagnostiziert. Giani- 
Hier steht eine Anzeige.

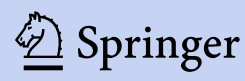


Tab. 2 Fallsterblichkeiten 2020 im Vergleich

\begin{tabular}{|l|l|l|l|}
\hline & SARS-CoV-2 & Influenza 2020 & Norovirus 2020 \\
\hline Positiv & 162 & 243 & 126 \\
\hline Positiv verstorben & 47 & 6 & 2 \\
\hline Fallsterblichkeit (+) & $29,0 \%$ & $2,5 \%$ & $1,6 \%$ \\
\hline Negativ & 1033 & 900 & 856 \\
\hline Negativ verstorben & 61 & 43 & 49 \\
\hline Fallsterblichkeit (-) & $5,9 \%$ & $4,8 \%$ & $6,9 \%$ \\
\hline Fallsterblichkeiten Klinikum gesamt $2,0 \%$ & & \\
\hline
\end{tabular}

Tab. 3 Fallsterblichkeit („,case fatality proportion “[CFP]) SARS-CoV-2 - Klinikum Fürth

\begin{tabular}{|c|c|c|c|c|c|c|}
\hline \multirow{4}{*}{$\begin{array}{l}\text { Alters- } \\
\text { gruppe }\end{array}$} & \multicolumn{3}{|l|}{ Positiv } & \multicolumn{3}{|l|}{ Negativ } \\
\hline & \multirow{3}{*}{$\begin{array}{l}\text { Anzahl } \\
162\end{array}$} & \multirow{3}{*}{$\%$ Positiv } & \multirow{3}{*}{$\begin{array}{l}\text { CFP } 29 \% \\
\text { Verstorben } \\
47\end{array}$} & \multirow{3}{*}{$\begin{array}{l}\text { Anzahl } \\
1033\end{array}$} & \multirow{3}{*}{$\%$ Negativ } & \multirow{3}{*}{$\begin{array}{l}\text { CFP 5,9\% } \\
\text { Verstorben } \\
61\end{array}$} \\
\hline & & & & & & \\
\hline & & & & & & \\
\hline $0-9$ & 1 & 0,6 & 0 & 7 & 0,7 & 1 \\
\hline $10-19$ & 0 & 0,0 & 0 & 17 & 1,6 & 1 \\
\hline $20-29$ & 0 & 0,0 & 0 & 46 & 4,5 & 3 \\
\hline $30-39$ & 1 & 0,6 & 0 & 54 & 5,2 & 3 \\
\hline $40-49$ & 7 & 4,3 & 0 & 69 & 6,7 & 6 \\
\hline $50-59$ & 7 & 4,3 & 0 & 110 & 10,6 & 9 \\
\hline $60-69$ & 24 & 14,8 & 3 & 177 & 17,1 & 10 \\
\hline 70-79 & 37 & 22,8 & 11 & 257 & 24,9 & 11 \\
\hline$\geq 80$ & 85 & 52,5 & 33 & 296 & 28,7 & 17 \\
\hline $80-89$ & 71 & 43,8 & 28 & 251 & 24,3 & 15 \\
\hline$\geq 90$ & 14 & 8,6 & 5 & 45 & 4,4 & 2 \\
\hline
\end{tabular}

Tab. 4 Fallsterblichkeit („case fatality proportion“ [CFP]) Influenza A/B 2019 - Klinikum Fürth

\begin{tabular}{|c|c|c|c|c|c|c|}
\hline \multirow{4}{*}{$\begin{array}{l}\text { Alters- } \\
\text { gruppe }\end{array}$} & \multicolumn{3}{|l|}{ Positiv } & \multicolumn{3}{|l|}{ Negativ } \\
\hline & & & \multirow{3}{*}{$\begin{array}{l}\text { CFP 5,7\% } \\
\text { Verstorben } \\
19\end{array}$} & \multirow{3}{*}{$\begin{array}{l}\text { Anzahl } \\
1115\end{array}$} & \multirow{3}{*}{$\%$ Negativ } & \multirow{3}{*}{$\begin{array}{l}\text { CFP 4,9\% } \\
\text { Verstorben } \\
55\end{array}$} \\
\hline & \multirow{2}{*}{$\begin{array}{l}\text { Anzahl } \\
336\end{array}$} & \multirow[t]{2}{*}{$\%$ Positiv } & & & & \\
\hline & & & & & & \\
\hline $0-9$ & 78 & 23,2 & 1 & 335 & 30,0 & 0 \\
\hline 10-19 & 11 & 3,3 & 0 & 33 & 3,0 & 0 \\
\hline $20-29$ & 13 & 3,9 & 0 & 19 & 1,7 & 0 \\
\hline $30-39$ & 10 & 3,0 & 0 & 25 & 2,2 & 0 \\
\hline $40-49$ & 14 & 4,2 & 1 & 22 & 2,0 & 2 \\
\hline $50-59$ & 33 & 9,8 & 1 & 61 & 5,5 & 0 \\
\hline $60-69$ & 23 & 6,8 & 1 & 127 & 11,4 & 6 \\
\hline 70-79 & 61 & 18,2 & 4 & 209 & 18,7 & 15 \\
\hline$\geq 80$ & 93 & 27,7 & 11 & 284 & 25,5 & 32 \\
\hline $80-89$ & 73 & 21,7 & 9 & 215 & 19,3 & 18 \\
\hline$\geq 90$ & 20 & 6,0 & 2 & 69 & 6,2 & 14 \\
\hline
\end{tabular}

colo et al. berichten über ein Maximum der bestätigten CFP von $30,6 \%$ bei über 80-Jährigen in Deutschland [9]. In der vorliegenden Studie bestätigte sich dieses Maximum bei über 80 -Jährigen mit einer CFP von 38,8\% (33/85). Die Influenza-CFP waren deutlich niedriger, erreichten aber auch bei über 80 -Jährigen
Spitzenwerte von 12,5\% und erniedrigten sich wieder bei über 90-Jährigen bei allen 3 Infektionserkrankungen. Auch die verstorbenen Noroviruspatienten fanden sich ausschließlich in den genannten Altersklassen.

Von den verstorbenen SARS-CoV-2positiven Patienten wurden nur 11 inva- siv beatmet, da $83,3 \%$ eine intensivmedizinische Behandlung abgelehnt hatten (DNR/DNI). Insgesamt wurden 87,0\% (141/162) aller SARS-CoV-2-Patienten auf Normalstation behandelt. Im Kontext dieser Zahlen und des demographischen Wandels sollten, neben der vielfach geführten Diskussion zu intensivmedizinischen Vorhaltungen und palliativmedizinischen Konzepten, vor allem die Aspekte der Infektionen im Alter und der stationären Behandlungskapazitäten in den Vordergrund gerückt werden [7, 10]. Auch allgemeinverfügte Besuchsverbote in Alten- und Pflegeheimen sollten bei diesen Zahlen überdacht werden.

Da alle 3 Infektionserkrankungen saisonal gehäuft vorkommen, ist anzunehmen, dass die jahreszeitlichen Hospitalisationen weiter zunehmen und die bereits bekannten Engpässe in Kliniken, die zu Zwangsbelegungen durch den Rettungsdienst führen, sich weiter verstärken werden [23].

In der besonders schweren Influenzasaison 2017/18 war laut RKI [27] die Hospitalisierungsrate mit $17 \%$ niedriger als in der Saison 2018/19 mit 22\% [28]. Die Absolutzahlen an Hospitalisierungen für 2017/18 waren mit 60.000 aber ca. 20.000 über denen der Saison 2018/19. Da die bevölkerungsbezogene Prävalenz von SARS-CoV-2 auf ca. $1 \%$ geschätzt wird, sollten, unter Annahme eines saisonalen Verlaufs, parallel zu Influenza und Norovirus proaktiv stationäre Behandlungskapazitäten saisonal eingeplant werden [29].

Die Hospitalisation beträgt für SARSCoV-2 in dieser Studie 19,7\% und addiert sich damit zur Hospitalisierungsrate für Influenza, die mit 20,3\% einen ähnlichen Wert für 2020 ergab und hauptsächlich ältere Patienten betraf. Aus Kliniksicht lag die stationäre Prävalenz für SARSCoV-2 mit 4,1\% deutlich über der von Influenza- und Norovirusinfektionen (- Tab. 1). Im internationalen Vergleich lag Deutschland Anfang April mit 10\% Hospitalisationen aufgrund von SARSCoV-2 niedrig verglichen mit Frankreich (40\%) oder Spanien (50\%) [22]. Allerdings muss bei diesen Zahlen beachtet werden, dass der Anteil der Hospitalisierungen in Regionen, in denen insgesamt wenig Tests durchgeführt werden oder 


\begin{tabular}{|c|c|c|c|c|c|c|}
\hline \multirow{4}{*}{$\begin{array}{l}\text { Alters- } \\
\text { gruppe }\end{array}$} & \multicolumn{3}{|l|}{ Positiv } & \multicolumn{3}{|l|}{ Negativ } \\
\hline & & & CFP $2,5 \%$ & & & CFP $4,8 \%$ \\
\hline & Anzahl & $\%$ Positiv & Verstorben & Anzahl & $\%$ Negativ & Verstorben \\
\hline & 243 & & 6 & 900 & & 43 \\
\hline $0-9$ & 93 & 38,3 & 0 & 306 & 34,0 & 0 \\
\hline $10-19$ & 18 & 7,4 & 0 & 38 & 4,2 & 1 \\
\hline $20-29$ & 14 & 5,8 & 0 & 16 & 1,8 & 0 \\
\hline $30-39$ & 14 & 5,8 & 0 & 37 & 4,1 & 0 \\
\hline $40-49$ & 17 & 7,0 & 0 & 40 & 4,4 & 0 \\
\hline $50-59$ & 17 & 7,0 & 0 & 60 & 6,7 & 2 \\
\hline $60-69$ & 18 & 7,4 & 0 & 84 & 9,3 & 3 \\
\hline 70-79 & 20 & 8,2 & 2 & 135 & 15,0 & 11 \\
\hline$\geq 80$ & 32 & 13,2 & 4 & 184 & 20,4 & 26 \\
\hline $80-89$ & 26 & 10,7 & 4 & 154 & 17,1 & 22 \\
\hline$\geq 90$ & 6 & 2,5 & 0 & 30 & 3,3 & 4 \\
\hline
\end{tabular}

Tab. 6 Fallsterblichkeit („case fatality proportion“ [CFP]) Norovirus 2019/2020 - Klinikum Fürth

\begin{tabular}{|c|c|c|c|c|c|c|}
\hline \multirow{4}{*}{$\begin{array}{l}\text { Alters- } \\
\text { gruppe }\end{array}$} & \multicolumn{3}{|l|}{ Positiv } & & \multirow[t]{2}{*}{ Negativ } & \multirow[b]{2}{*}{ CFP 6,9\% } \\
\hline & & & CFP 1,6 \% & & & \\
\hline & Anzahl & $\%$ Positiv & Verstorben & Anzahl & $\%$ Negativ & Verstorben \\
\hline & 125 & & 2 & 856 & & 59 \\
\hline $0-9$ & 52 & 41,6 & 0 & 154 & 18,0 & 0 \\
\hline 10-19 & 8 & 6,4 & 0 & 50 & 5,8 & 0 \\
\hline $20-29$ & 8 & 6,4 & 0 & 20 & 2,3 & 0 \\
\hline $30-39$ & 9 & 7,2 & 0 & 18 & 2,1 & 0 \\
\hline $40-49$ & 2 & 1,6 & 0 & 17 & 2,0 & 0 \\
\hline $50-59$ & 6 & 4,8 & 0 & 57 & 6,7 & 1 \\
\hline $60-69$ & 4 & 3,2 & 0 & 82 & 9,6 & 8 \\
\hline 70-79 & 7 & 5,6 & 1 & 150 & 17,5 & 10 \\
\hline$\geq 80$ & 29 & 23,2 & 1 & 308 & 36,0 & 40 \\
\hline $80-89$ & 23 & 18,4 & 0 & 232 & 27,1 & 31 \\
\hline$\geq 90$ & 6 & 4,8 & 0 & 76 & 8,9 & 9 \\
\hline
\end{tabular}

sich die Tests auf hospitalisierte Patienten konzentrieren wie in unserer Studie bei Noroviruspatienten, deutlich höher liegen können. Im internationalen Vergleich war auf regionaler Ebene die Lombardei (Italien) mit 132,5/100.000 stationären Patienten am stärksten betroffen. Daten aus der COVID-19-Surveillance des Centers for Disease Control (CDC) zeigen für die USA eine Gesamtrate an hospitalisierten Patienten von 67,9/100.000. Für Stadt- und Landkreis Fürth ergab sich mit 66,1/100.000 ein ähnlicher Wert, wobei die Gruppe der Menschen über 65 Jahre $(\bullet A b b .1 b)$, wie auch in anderen Studien z.B. für die USA mit 214,4/100.000, den höchsten Wert erreichte [3]. menhang mit COVID-19 verstorbenen Patienten [4].

Laut demographischer Daten des RKI waren $1,8 \%$ der Erkrankten jünger als 10 Jahre und nur 4,2\% 10-19 Jahre alt [6, 29]. Die Daten des Gesundheitsamts Fürth zeigen insgesamt 23 infizierte Kinder zwischen 0-14 Jahre, was einer Rate von $2,8 \%$ entspricht. Im gesamten $\mathrm{Be}$ obachtungszeitraum wurde lediglich ein positiv getesteter Säugling stationär aufgenommen, dessen Aufnahmeindikation allerdings nicht die SARS-CoV-2-Infektion war. Diese Daten decken sich mit einer deutschlandweiten Untersuchung 128 hospitalisierter Kinder aus $66 \mathrm{Kli}-$ niken [1]. 85 Kinderkliniken meldeten aktiv, keine Kinder mit COVID-19 behandelt zu haben. 16 Patienten mussten intensivmedizinisch betreut werden, ein Säugling ist im Zusammenhang mit Covid-19 verstorben. Auch Prof. Debatin zeigt in der Eltern-Kind-COVID-19 Studie aus Baden-Württemberg, dass Kinder weniger als ein Drittel der Betroffenen stellten und somit nicht nur seltener an COVID-19 erkrankten, sondern auch seltener mitSARS-CoV-2 infiziert zu sein schienen [5]. Die aktuellen Daten über die Erkrankungshäufigkeit und die potenzielle Infektiosität von Kindern bezogen auf SARS-CoV-2 sind in Diskussion und werfen vermehrt Forschungsfragen auf $[11,13,15]$.

\section{Limitationen der Studie}

Der Vergleich der 3 Infektionserkrankungen zeigt zwar Parallelen die Erkrankungshäufigkeiten bei zunehmendem Alter betreffend, aber auch massive Unterschiede in der Altersverteilung der hospitalisierten Patienten, die für SARSCoV-2 keine Häufung im Kindes- und Jugendalter ergaben (• Abb. 1b; $[16,20]$ ).

Auch die Daten des CDC zeigen, dass 0 - bis 17-Jährige deutlich weniger häufig wegen SARS-CoV-2 hospitalisiert werden mussten als wegen Influenza [3], da die überwiegende Mehrzahl der klinischen Verläufe bei Kindern mild und unspezifisch ist $[8,17]$ und eine sehr geringe Letalität aufweist. Das CDC berichtete über 9 verstorbene Kinder im Alter bis 14 Jahre bei insgesamt 37.308 in Zusam-
Es handelt sich um die Auswertung von Patientendaten einer Kommune und dessen umfassenden Notfallversorgers, sodass eine Übertragung der Ergebnisse auf nationaler Ebene, trotz repräsentativer Altersstruktur der Kommune, nur teilweise möglich ist.

Die Indikationsstellung zur PCR-Testung und Qualität der Testverfahren wurden nicht weiter analysiert.

\section{Schlussfolgerungen}

Die Influenzaübersterblichkeit 2017/2018 hatte bisher nicht zu einer Veränderung der medizinischen Infrastruktur geführt $[14,30]$. Durch das öffentliche Interesse an COVID-19 ergeben sich Chancen auf eine Neuausrichtung im Bereich der am- 
bulanten und stationären Versorgung. Es sollten außerdem auch die negativ getesteten Infektionserkrankungen, die sich derzeit jeder Statistik entziehen, $\mathrm{zu}$ den benötigten Versorgungsstrukturen miteinkalkuliert werden, da deren CFP die der positiv getesteten teilweise sogar übersteigen. Mit SARS-CoV-2 hat sich eine weitere Infektionserkrankung in der Bevölkerung etabliert, die neben den Intensivkapazitäten zusätzlichen Ressourcenaufwand, vor allem auf Normalstationsebene und im Bereich der Notaufnahmen, verursachen wird. Patienten, die sich in der Herbst-/ Wintersaison mit respiratorischer Insuffizienz, Zeichen eines Atemwegsinfekts, grippalen oder gastrointestinalen Symptomen vorstellen, werden, um erneute Ausbruchssituationen und Übersterblichkeiten $\mathrm{zu}$ vermeiden, einen deutlichen Mehraufwand an Ressourcen benötigen [32]. Screening- und Teststrategien, Isolationskonzepte sowie sichere Prozessabläufe sollten bereits jetzt entwickelt werden. Ein besonderer Fokus in der Versorgung gilt der Altersmedizin, wohingegen die geringe Betroffenheit der Kinder weitere Forschungsfragen aufwirft. Antworten darauf könnten dann das Bild zu SARS-CoV-2 vervollständigen.

\section{Fazit für die Praxis}

\section{- Ambulante SARS-CoV-2-positive}

Patienten sind meist 35-59 Jahre alt; $>50 \%$ der Hospitalisierten $\geq 80$ Jahre alt.

- Die Altersverteilung von SARS-CoV2-Patienten unterscheidet sich zu Influenza/Norovirus; kein Krankheitsgipfel bei Kleinkindern. Kinder zeigen kaum SARS-CoV-2-Infektionen (23 von $824 ; 2,8 \%$ ) mit meist mildem Verlauf.

- Die Hospitalisationsrate in Fürth ist bei Influenza- $(20,3 \%)$ und SARSCoV-2-Positiven (19,7\%) gleich, die Fallsterblichkeit (CFP) hospitalisierter SARS-CoV-2-Patienten signifikant höher. Insgesamt überlebten 71,0\% aller stationären SARS-CoV-2-Patienten, 2 von 3 der > 90-Jährigen. SARS-CoV-2-Patienten lehnten bei
Aufnahme intensivmedizinische Maßnahmen mit invasiver Beatmung ab, $83 \%$ die Reanimation.

- Die CFP negativ getesteter Verdachtspatienten lag bei Noroviruspatienten deutlich über der positiv getesteter (6,9\% vs. 1,6\%); die Streubreite aller testnegativen Infektionspatienten $(4,8-6,9 \%)$ war ähnlich (Krankenhausmortalität 2,1\%).

\section{Korrespondenzadresse}

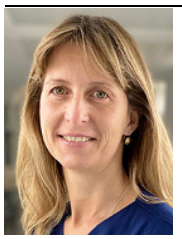

Dr. M. V. Karg

Zentrale Notaufnahme, Klinikum Fürth Jakob-Henle-Str. 1, 90766 Fürth, Deutschland karg.marina@gmail.com

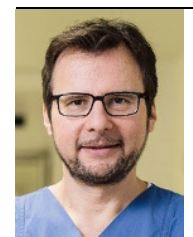

Prof. Dr. H. Dormann Zentrale Notaufnahme, Klinikum Fürth Jakob-Henle-Str. 1, 90766 Fürth, Deutschland harald.dormann@ klinikum-fuerth.de

Danksagung. Wir bedanken uns für die hervorragende Zusammenarbeit zwischen dem Landratsamt Abteilung für Gesundheit Fürth und dem Bayerischen Landesamt für Statistik sowie den jeweiligen Mitarbeitern, die diese Studie ermöglicht haben. Wir danken den Mitarbeitern des medizinischen Controllings und des betriebswirtschaftlichen Controllings des Klinikums Fürth für die professionelle Teamarbeit und Unterstützung während der Studie, insbesondere Herrn Steffen Dietz, Herrn Dieter Kolb und Herrn Riko Schmidt. Ein besonderer Dank geht an die Mitarbeiter der ZNA-Studienzentrale des Klinikums Fürth für die Unterstützung bei der Datenerhebung, Auswertung und Erstellung von Graphiken, insbesondere Frau Anne Wagner. Darübe hinaus danken wir dem medizinischen Direktor und Pandemiebeauftragten des Klinikums Fürth, Herrn Dr. med. Manfred Wagner, für die Motivation und hilfreichen Anregungen.

\section{Einhaltung ethischer Richtlinien}

Interessenkonflikt. M. V. Karg, B. Alber, C. Kuhn, K. Bohlinger, M. Englbrecht und H. Dormann geben an, dass kein Interessenkonflikt besteht.

Für diesen Beitrag wurden von den Autoren keine Studien an Menschen oder Tieren durchgeführt. Für die aufgeführten Studien gelten die jeweils dort angegebenen ethischen Richtlinien. Ein positives Votum zur Durchführung der Studie der Ethikkommission der Friedrich-Alexander-Universität Erlangen-Nürnberg liegt vor.

\section{Literatur}

1. Armann J, Diffloth N, Simon A et al (2020) Hospital admission in children and adolescents with COVID-19-early results from a national survey conducted by the German Society for Pediatric Infectious Diseases (DGPI). Dtsch Arztebl Int 117:373-374. https://doi.org/10.3238/arztebl. 2020.0373

2. Briel C, Wilhelm K, Heller J (2016) Krankenhausmortalität und -morbidität bei 448 konsekutiven Patienten mit Norovirusinfektion. Z Gastroenterol. https://doi.org/10.1055/s-0036-1586924

3. Center for Disease Control (2020) Covid-19 surveillance. https://www.cdc.gov/coronavirus/ 2019-ncov/covid-data/covidview/index.html. Zugegriffen:28. Mai 2020

4. Center for Disease Control (2020) Weekly updates by select demographic and geographic characteristics provisional death counts for coronavirus disease (COVID-19). https://www.cdc.gov/nchs/ nvss/vsrr/covid_weekly/index.htm. Zugegriffen: 12. Mai 2020

5. Debatin K (2020) Vorläufige Ergebnisse der Eltern-Kind Covid-19-Studie in Baden-Württemberg. https://www.klinikum.uni-heidelberg.de/ newsroom/vorlaeufige-ergebnisse-der-elternkind-covid-19-studie-in-baden-wuerttemberg/. Zugegriffen: 23. Juni 2020

6. Deutsche Akademie für Kinder- und Jugendmedizin (DAKJ) e.V. (2020) Wiederaufnahme der Betreuung von Kindern im Vorschulalter Stellungnahme der Kommission Frühe Betreuung und Kindergesundheit der DAKJ. https://www.dakj. de/allgemein/wiederaufnahme-der-betreuung von-kindern-im-vorschulalter/. Zugegriffen: 3. Juni 2020

7. Deutsche Gesellschaft für Palliativmedizin (2020) Empfehlungen der DGP zu COVID-19 (Coronavirus SARS-CoV-2). https://www.dgpalliativmedizin. de/neuigkeiten/empfehlungen-der-dgp.html. Zugegriffen: 3. Juni 2020

8. Dong Y, Mo X, Hu Y (2020) Epidemiology of COVID19 among children in China. Pediatrics. https://doi org/10.1542/peds.2020-0702

9. Gianicolo E, Riccetti N, Blettner M, Karch A (2020) Epidemiological measures in the context of the COVID-19 pandemic. Dtsch Arztebl Online. https:// doi.org/10.3238/arztebl.2020.0336

10. Gosch M, Singler K, Kwetkat A, Heppner HJ (2020) Geriatrie in Zeiten von Corona. Z Gerontol Geriatr 53:228-232. https://doi.org/10.1007/s00391020-01725-2

11. Gudbjartsson DF, Helgason A, Jonsson $\mathrm{H}$ et al (2020) Spread of SARS-CoV-2 in the Icelandic population. NEngl J Med. https://doi.org/10.1056/ NEJMoa2006100

12. Johns Hopkins Universität (2020) COVID-19 dashboard by the Center for Systems Science and Engineering. https://gisanddata.maps. arcgis.com/apps/opsdashboard/index.html\#/ bda7594740fd40299423467b48e9ecf6. Zugegriffen: 26. Mai 2020

13. Jones $T$, Mühlemann $B$, Veith $B$ et al An analysis of SARS-CoV-2 viral load by patient age. https://virologie-ccm.charite.de/fileadmin/ user_upload/microsites/m_cc05/virologie-ccm/ dateien_upload/Weitere_Dateien/Charite_SARSCoV-2_viral_load_2020-06-02.pdf. Zugegriffen: 03. Juni 2020

14. Kraef $C$, van der Meirschen $M$, Wichmann D et al (2019) Management der saisonalen Influenza 2017/2018: Erfahrungen an einem deutschen Universitätsklinikum. Bundesgesundheits- 
blatt Gesundheitsforschung Gesundheitsschutz 62:870-880. https://doi.org/10.1007/s00103 019-02976-0

15. Lavezzo E et al (2020) Suppression of COVID-19 outbreak in the municipality of Vo, Italy. medRxiv. https://doi.org/10.1101/2020.04.17.20053157

16. Lian J, Jin X, Hao S et al (2020) Analysis of epidemiological and clinical features in older patients with coronavirus disease 2019 (COVID-19) outside Wuhan. Clin Infect Dis. https://doi.org/10. 1093/cid/ciaa242

17. Ludvigsson J (2020) Systematic review of COVID-19 in children shows milder cases and a better prognosis than adults. Acta Paediatr 109:1088-1095

18. Meier F, Bauer K, Schöffski O et al (2016) Zur Ökonomie ambulanter Notaufnahmepatienten: Untersuchung der Deckungsbeiträge in Abhängigkeit von Dringlichkeitskategorien, Leitsymptomen und Diagnosen. Notfall Rettungsmed 19:33-40. https://doi.org/10.1007/s10049-015-0054-4

19. Mizumoto K, Kagaya K, Zarebski A, Chowell G (2020) Estimating the asymptomatic proportion of coronavirus disease 2019 (COVID-19) cases on board the Diamond Princess cruise ship, Yokohama, Japan, 2020. Euro Surveill. https://doi. org/10.2807/1560-7917.ES.2020.25.10.2000180

20. Onder G, Rezza G, Brusaferro S (2020) Case-fatality rate and characteristics of patients dying in relation to COVID-19 in Italy. JAMA. https://doi.org/10. 1001/jama.2020.4683

21. ORF Tirol News (2020) Paznaun und St. Anton weiter in Quarantäne. https://tirol.orf.at/stories/ 3043535. Zugegriffen: 28. Mai 2020

22. Busse R (2020) COVID-19: Datenbankzu Patienten, die in 15 europäischen Ländern stationär und im Krankenhaus behandelt werden - täglich aktualisiert. https://www.mig.tu-berlin.de/fileadmin/ a38331600/sonstiges/COVID-19-STATS_27052200.pdf.Zugegriffen:29. Mai 2020

23. Rittberg W, Pfüger P, Ledwoch J et al (2020) Forced centralized allocation of patients to "closed" emergency departments-data from a German city. Dtsch Arztebl Int. https://doi.org/10.3238/ arztebl.2020.0465

24. RKI Ratgeber Norovirus-Gastroenteritis. https:// www.rki.de/DE/Content/Infekt/EpidBull/ Merkblaetter/Ratgeber_Noroviren.html. Zugegriffen: 3 . Juni 2020

25. Robert Koch Institut (2020) Täglicher Lagebericht des RKI zur Coronavirus-Krankheit2019 (COVID-19) vom 07.05.2020. https:// www.rki.de/DE/Content/InfAZ/N/Neuartiges Coronavirus/Situationsberichte/2020-05-07-de. pdf?__blob=publicationFile. Zugegriffen: 14 . Mai 2020

26. Robert Koch Institut (2020) Erste Ergebnisse zum Verlauf der Grippewelle in der Saison 2019/20: Mit 11 Wochen vergleichsweise kürzere Dauer und eine moderate Anzahl an Influenza-bedingten Arztbesuchen. https://www.rki.de/DE/Content/ Infekt/EpidBull/Archiv/2020/16/Art_02.html; jsessionid=F7CF1AEEE199FA3FAE89E5C930E1F812. internet051?nn=2370434. Zugegriffen: 3. Juni 2020

27. Robert Koch Institut Bericht zur Epidemiologie der Influenza in Deutschland Saison 2017/18. https://influenza.rki.de/Saisonberichte/2017.pdf. Zugegriffen:27. Mai 2020

28. Robert Koch Institut Bericht zur Epidemiologie der Influenza in Deutschland Saison 2018/19. https://influenza.rki.de/Saisonberichte/2018.pdf. Zugegriffen: 27. Mai 2020

29. Robert Koch Institut SARS-CoV-2 Steckbrief zur Coronavirus-Krankheit-2019 (COVID-
19). https://www.rki.de/DE/Content/InfAZ/ N/Neuartiges_Coronavirus/Steckbrief.html\# doc13776792bodyText2.Zugegriffen:7. Mai 2020

30. Sachverständigenrat (2018) Bedarfsgerechte Steuerung der Gesundheitsversorgung Gutachten. https://www.svr-gesundheit.de/fileadmin/ user_upload/Gutachten/2018/SVR-Gutachten_ 2018_WEBSEITE.pdf.Zugegriffen:23. Juni 2020

31. Streeck H, Schulte B, Kuemmerer B et al (2020) Infection fatality rate of SARS-CoV-2 infection in a German community with a super-spreading event. medRxiv. https://doi.org/10.1101/2020.05. 04.20090076

32. Wennmann DO, Dlugos CP, Hofschröer A et al (2020) Umgang mit COVID-19 in der Notaufnahme: Erfahrungsbericht der interdisziplinären Notaufnahme des Universitätsklinikums Münster. Med Klin Intensivmed Notfallmed 115:380-387. https://doi.org/10.1007/s00063-020-00693-0

33. WHO Coronavirus disease (COVID-19) Situation Report-127. https://www.who.int/ docs/default-source/coronaviruse/situationreports/20200526-covid-19-sitrep-127.pdf? sfvrsn=7b6655ab_8.Zugegriffen:26. Mai 2020

34. SORA Institut (2020) Spread of SARS-CoV-2 in Austria PCR tests in a representative sample Study report. https://www.sora.at/fileadmin/ downloads/projekte/Austria_Spread_of_SARSCoV-2_Study_Report.pdf. Zugegriffen: 23. Juni 2020

35 . Verity R et al (2020) Estimates of the severity of coronavirus disease 2019: a model-based analysis. Lancet. https://doi.org/10.1016/S14733099(20)30243-7
MED UPDATE SEMINARE 2022

Intensiv Update 2022

14. Intensivmedizin-Update-Seminar 23.-24. September 2022 Köln und Livestream

Wiss. Leitung:

Prof. Dr. Tobias Welte, Hannover

Prof. Dr. Stefan Kluge, Hamburg

Prof. Dr. Uwe Janssens, Eschweiler Prof. Dr. Frank Tacke, Berlin

Unter der Schirmherrschaft DGIM, DGIIN, DIVI, ÖGIAIN

www.intensiv-update.com

Auskunft für alle Update-Seminare: med update $\mathrm{GmbH}$ www.med-update.com

Tel.: 0611 - 736580

info@med-update.com

\section{medupdate}

